\title{
Overweight and obesity in children: a comparison of the views of general practitioners and parents
}

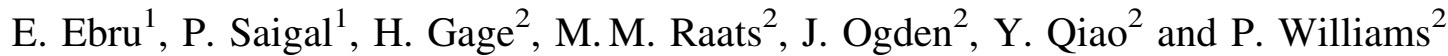 \\ ${ }^{1}$ King's College London, School of Medicine, Hodgkin Building, Guy's Campus, London SE1 1UL, UK and ${ }^{2}$ Food, \\ Consumer Behaviour and Health Research Centre, University of Surrey, Guildford GU2 7XH, UK
}

The growing problem of childhood overweight and obesity has raised questions about optimal management and how responsibility for reversing the trends is divided between government, families, schools, industry and the health sector. At the level of the individual child, action depends on recognition of overweight and obesity. A survey was sent to all general practitioners (GP) in one Primary Care Trust (Surrey) in England and to all parents in one primary school in south London to investigate their views about the causes, consequences and management of childhood overweight and obesity. Questionnaires also contained fourteen (seven boys, seven girls) validated images representing standard percentile BMI for healthy children ${ }^{(1)}$. Respondents were asked to state whether they thought each image was underweight, acceptable weight, overweight or obese. Differences between GP and parents were analysed using $\chi^{2}$ tests.

Of 583 questionnaires mailed to GP $184(31.6 \%)$ were returned. The median age of respondents was 45 years and $55.4 \%$ were women (compared with $43.4 \%$ in the whole sample). Responding GP were more likely to come from larger practices (five or more partners). A total of 135 parents returned completed questionnaires, their median age was 40 years, $89.6 \%$ were women and $83 \%$ reported having completed further or higher education. The GP and parent samples were similar in relation to ethnicity $(85 \%$ and $83 \%$ respectively were white), but more GP than parents considered themselves to be overweight or obese (33\% v. 17\%).

Approximately all GP and parents ( $>95 \%$ in both groups) recognised that inadequate physical activity was a cause of overweight or obesity in children. Slightly lower percentages identified poor diet (90) and 'junk food advertisement' (80) as contributory factors. GP were significantly more likely than parents to agree that peer pressure $(48.6 \% v .31 .6 \% ; P=0.001)$, low family income $(41.4 \%$ v. $29.5 \%$; $P=0.0003)$ and (marginally) genetics $(45.2 \% v .36 .5 \% ; P=0.10)$ were causes of overweight or obesity in children. GP and parents agreed unanimously that parents should be involved in the management of childhood overweight and obesity, but parents were significantly more likely than GP to think that GP should be involved $(86.4 \% v .73 .3 \% ; P=0.0004)$, and GP were significantly more likely than parents to agree that school nurses $(97.9 \%$ v. $83.5 \% ; P=0.0001)$ and children $(95.8 \%$ v. $87.9 \% ; P=0.001)$ should be active participants. Both groups tended to overstate the extent of overweight and obesity (Table). More-highly-educated parents were more likely to give correct assessments (data not shown).

\begin{tabular}{|c|c|c|c|c|c|c|c|c|c|c|c|c|c|c|}
\hline & \multicolumn{7}{|c|}{ Boys } & \multicolumn{7}{|c|}{ Girls } \\
\hline & 1 & 2 & 3 & 4 & 5 & 6 & 7 & 1 & 2 & 3 & 4 & 5 & 6 & 7 \\
\hline Correct answer & OK & OK & OK & $\mathrm{OK}$ & $\mathrm{OK}$ & Over & Obese & Under & OK & $\mathrm{OK}$ & OK & OK & Over & $\overline{\text { Obese }}$ \\
\hline$\%$ GPs correct & 40.2 & 96.2 & 94.1 & 29.2 & 75.5 & 71.4 & 95.1 & 91.9 & 54.3 & 95.1 & 74.5 & 29.0 & 49.5 & 91.8 \\
\hline$\%$ Parents correct & 30.2 & 94.2 & 96.4 & 40.2 & 85.5 & 64.8 & 92.0 & 89.1 & 51.8 & 95.7 & 86.2 & 29.5 & 59.4 & 89.9 \\
\hline Parents $v$. GPs: $P$ & 0.064 & NS & NS & 0.04 & 0.03 & NS & NS & NS & NS & NS & 0.01 & NS & 0.08 & NS \\
\hline Incorrect answers & Under & & & Over & Obese & & & & Under & & Over & Over & Obese & \\
\hline
\end{tabular}

OK, acceptable weight; Under, underweight; Over, overweight.

In response to a vignette describing an overweight child presenting at the GP surgery with a minor respiratory infection, $43.1 \%$ of GP and $24.0 \%$ of parents thought that the GP should take no action about the child's weight. GP identified lack of time (cited by $67.8 \%$ ), financial incentive $(36.3 \%)$, expertise $(32.1 \%)$ and other support systems $(82.2 \%)$ as barriers to weight management.

Awareness of overweight and obesity needs to be translated into action in order to prevent serious public health problems in the future. Differences in the perceptions of parents and GP about responsibility for addressing the problem of childhood overweight and obesity need to be reconciled if an effective strategy is to be implemented.

1. Truby H \&Paxton S (2002) Br J Clin Psychol 41, 185-203. 